\title{
Cooperative Infrastructure and Spectrum Sharing in Heterogeneous Mobile Networks
}

\author{
Lorela Cano, Antonio Capone, Senior Member, IEEE, Giuliana Carello, Matteo Cesana, Member, IEEE, \\ and Mauro Passacantando
}

\begin{abstract}
To accommodate the ever-growing traffic load and bandwidth demand generated by mobile users, Mobile Network Operators (MNOs) need to frequently invest in high spectral efficiency technologies and increase their hold of spectrum resources; MNOs have then to weigh between building individual networks or entering into network and spectrum sharing agreements. We address here the problem of Radio Access Network (RAN) and spectrum sharing in $4 \mathrm{G}$ mobile networks by focusing on a case when multiple MNOs plan to deploy small cell Base Stations in a geographical area in order to upgrade their existing network infrastructure. We propose two cooperative game models (with and without transferable utility) to address the proposed problem: for given network (user throughput, MNO market and spectrum shares) and economic (coalition cost, mobile data pricing model) settings, the proposed models output a cost division policy that guarantees coalition (sharing agreement) stability.
\end{abstract}

Index Terms-RAN sharing, spectrum sharing, 4G, cooperative games, transferable utility, non-transferable utility, core, nucleolus.

\section{INTRODUCTION}

$\mathbf{T}$ HE mobile network ecosystem is intrinsically competitive as Mobile Network Operators (MNOs) are selfinterested entities. However, expensive technology upgrades to support user demand [1], revenues decline [2], regulators intervention [3] and communities health/environmental concerns are pushing competing operators to cooperate and share their networks. Nevertheless, cost reduction remains the main driver for network sharing: The mobile market is characterized by high upfront cost for acquiring spectrum licenses and deploying and operating the network infrastructure, which is particularly heavy on new-entrants [3]. Nowadays, MNOs need to invest in high spectral efficiency mobile technologies such as LTE-A and 5G and, in particular, to increase their hold of spectrum resources in order to accommodate the exponentially growing demand for mobile data services [1]. To the high upgrade upfront cost amounts the decrease in revenues, strongly due to Over The Top (OTT) applications replacing MNOs main revenue resources such as voice and SMS [4]: but as investment in new technologies becomes little profitable, innovation is held back.

Network sharing agreements for greenfield network roll-outs have become a means to reduce the high upfront infrastructure

This paper has been published in IEEE Journal on Selected Areas in Communications, vol. 34 (2016), pp. 2617-2629.

L.Cano, A.Capone, G.Carello and M.Cesana are with DEIB (Dipartimento di Elettronica, Informazione e Bioingegneria), Politecnico di Milano, Italy.

M.Passacantando is with Dipartimento di Informatica, Università di Pisa, Italy. cost and, when spectrum sharing is allowed ${ }^{1}$, to boost the network capacity by aggregating spectrum resources.

Network sharing can encompass different parts of the network architecture in addition to having different geographical footprints [8,9]. Passive sharing (site and mast sharing), the most commonplace sharing alternative, is either mandated (or strongly encouraged) by regulators or voluntary adopted given the limited site availability, urban planning constraints and communities aesthetics and health concerns [9]-[11]. Up to date, several 50:50 joint ventures for $3 \mathrm{G} / 4 \mathrm{G}$ greenfield network deployments have been created; while most concern only sharing of the Radio Access Network (RAN) infrastructure, in some cases, also spectrum is shared $[6,11]$.

We address here the common spectrum network sharing scenario, as defined in the Third Generation Partnership Project (3GPP) specifications for network sharing [12]. Namely, we assume multiple MNOs offer their services through a single (shared) RAN while running individual core networks; "MNOs share the total spectrum obtained from pooling together their respective allocated spectrum portions while it is also possible for MNOs with no allocated spectrum to use the pooled spectrum" [12]. One viable option for implementing such scenario in $4 \mathrm{G}$ networks is Carrier Aggregation (CA), an LTEA standardized feature [13] that enables pooling together the spectrum allocated in different bands ${ }^{2}$.

In this work, we consider a set of MNOs with fixed market shares and individual spectrum licenses, which plan to upgrade their RAN by deploying small cell Base Stations (BSs) in order to improve the service provided to their users and thus increase their revenues. MNOs decide whether to upgrade their RAN by themselves or enter into a sharing agreement with other MNOs. If a set (all) of MNOs enters into a sharing agreement, that is, a coalition is created, we assume it will make use of all the aggregated spectrum resources of its members. We propose two cooperative game theory models, with and without transferable utility, to determine stable cost divisions for coalitions of MNOs entering sharing agreements. The proposed models are then leveraged to investigate several network (user throughput, market and spectrum shares) and

\footnotetext{
${ }^{1}$ In practice, both infrastructure and spectrum sharing viability are subject to national and regional/international regulation. Sharing and/or transferring of licensed spectrum is prohibited in most countries [5]. There are however examples of spectrum sharing: for instance, in Sweden, operator Tele2 is into a $3 \mathrm{G}$ license and network sharing agreement with TeliaSonora and it has entered a similar agreement for deploying a $4 \mathrm{G}$ network with Telenor [6], [7].

${ }^{2} 116$ operators have commercially launched LTE-A with CA [14]. CA will most likely be an enabler also for future generation networks given the $5 \mathrm{G}$ throughput targets.
} 
economic configurations (coalition cost, mobile data pricing model) which aim to represent realistic scenarios.

The main findings of this work are the following:

- If all MNOs contribute with spectrum resources, they prefer building a unique shared RAN due to the combined gain of spectrum aggregation and the cost reduction from sharing the network infrastructure; formally, this means that the reference cooperative game has a nonempty core, which makes the grand coalition preferable to any subcoalition.

- The stable division (among MNOs) of the shared network infrastructure cost depends on both network and economic settings: MNOs with a larger customer base should be accounted for a larger fraction of the network cost; instead, MNOs which contribute with a larger spectrum portion are "rewarded" by a lower cost fraction and, in some cases, not only they are exempted from such cost but also receive part of the other MNO individual revenues, which suggests a way to compensate them from most likely higher cost incurred when acquiring the spectrum license ${ }^{3}$.

- A trivial cost division based on the market share does not always guarantee stability; instead, the stable cost division selected by the nucleolus, which accounts also for the MNOs spectrum contribute, makes a better candidate for a cost division policy.

The manuscript is organized as follows: The literature review is presented in Section II. In Section III, we state the problem and define the coalitions cost and the proposed pricing model. The Transferable Utility (TU) and Non-Transferable Utility (NTU) cooperative models are introduced in Section IV. The simulation environment and the problem instances are described in Section V. Results obtained with the two cooperative models are analyzed in Section VI. In Section VII, we discuss some of the assumptions made and the applicability of the proposed models. Our concluding remarks are drawn in Section VIII.

\section{RELATED WORK}

Recent works on resource sharing deal mainly with operational aspects, such as scheduling of shared resources among multiple operators. [15] adopts the Generalized Processor Sharing principle to a multi-operator scheduler when operators agree a priori on their respective resource shares. In the same lines, [16] investigates the trade-off between fairness, that is, satisfying operators predefined resource shares and the achievable spectral efficiency by deviating from predefined resource shares. [17] introduces the SoftRAN architecture, which extends the concept of Software Defined Networks (SDN) to the RAN. A centralized scheduler for SoftRAN is proposed in [18]: the traffic of multiple operators is allocated over the 3D (time-frequency-space) resource grid with the objective of maximizing the total network utility. [19] proposes

\footnotetext{
${ }^{3}$ However, we do not account for the spectrum license cost here. Moreover the spectrum license cost does not depend only on the amount of bandwidth associated with the license but also on the spectrum band and and the time and place of the spectrum auction.
}

a 2-level radio resource scheduling (among MNOs, and for each MNO among its user flows) BS virtualization scheme.

Given the competitive and cooperative nature of resource sharing problems, many works resort to game theory: In [20], the problem of resource allocation in a shared network is formalized in two steps: the resource sharing among the operators, and the resource bargaining among the users and Mobile Virtual Network Operators of each operator. [21] investigates the sharing of different wireless access technologies. In particular, [22]-[25] tackle spectrum sharing problems. [22] models spectrum sharing among strategic operators in unlicensed bands as a noncooperative game. Instead, [23][25] deal with licensed spectrum. [25] also proposes a noncooperative game but takes the user perspective: assuming MNOs with individual spectrum resources aggregate their RANs, each user then independently selects its serving BS from the shared pool in order to maximize its individual data rate. [23] extends the concept of CA for limited-time sharing of excess spectrum among MNOs that own exclusive spectrum resources. Spectrum scheduling is carried out based on the Nash Bargaining Solution concept while a distributed algorithm is proposed for Bayesian coalition formation when the MNO decisions are made based on incomplete information. In [24], the inter-operator CA does not apply only to the MNOs' unutilized spectrum but, if profitable, MNOs can agree to share a portion of their individual spectrum between their own users and users of another MNO; the level of interference caused by the latter is controlled by means of a pricing mechanism. Such sharing scheme is limited only to two MNOs and the pairing of a set of MNOs for mutually sharing part of their spectrum is modeled as a stable roommate market.

As virtualization and SDN are expected to extend to wireless networks ([26], [27]), new architectures are anticipated ([18], [28], [29]). [28] and [29] envisage a "Network without Borders", as the pool of virtualized wireless resources which defies the current vertically-integrated mobile networks valuechain by introducing new players such as service/infrastructure providers and virtual operators. Inter-operator sharing is argued to be one of the key ingredients of such architecture. The idea is further elaborated in [29], where the focus is on novel spectrum management aspects.

Our work instead belongs to a complementary research branch, whose focus is on the strategic modeling of infrastructure and spectrum sharing. In particular, we consider the sharing of exclusive (licensed) MNO spectrum ${ }^{4}$.

On mid-to-long term joint decision making in the context of cellular network planning, [31] introduces a competition-aware network sharing framework which offers a trade-off between the cost benefit of sharing and the incentive for investing in next-generation technologies. While we address a greenfielddeployment of small cell BSs, [31] assumes operators will pool together their existing macro-cell RAN networks and make joint decisions on future changes to their aggregated RAN such as decommissioning, upgrading or adding new sites. Also in [32], a recent work by Kibilda et al., the shared network is

\footnotetext{
${ }^{4}$ The literature on dynamic spectrum access, cognitive/software radio etc. dealing with sharing of licensed and unlicensed spectrum [30] is not addressed here.
} 
created by pooling together the operators' individual network infrastructure and/or their respective licensed spectrum. This work compares the gains from infrastructure and spectrum sharing when adopted separately and combined (full sharing) on the basis of classical performance indicators such as throughput and coverage probability obtained by means of stochastic geometry models; such gains are shown to strongly depend on the spatial correlation of the individual network deployments and densities while infrastructure and spectrum sharing gains do not sum up as full sharing introduces a tradeoff between data rate and coverage.

However, [33]-[35] are the only works which bear explicit similarities with ours: they also tackle the strategic problem of coalition formation in the context of infrastructure and spectrum sharing and consider MNOs with fixed market shares and pre-allocated spectrum; nevertheless, these works resort to non-cooperative game theory. Moreover, players (MNOs) payoffs are expressed only in terms of network cost estimates and the coalition cost are split either uniformly among its members [33,34] or according to the Shapley value [35]. Instead, we propose more refined payoff models for the MNOs which accounts for both the MNO revenue (as a function of the average user rate perceived by users) and cost. Moreover, in the cooperative games proposed here the coalition cost is not divided a priori among member operators; albeit the way such cost is split determines the coalition stability.

In our previous work [36], we propose a Mixed Integer Linear Programming model to address an infrastructure sharing problem from a centralized/regulatory entity perspective. Instead, in this work and in [37], we take the perspective of the MNOs, which are self-interested entities, and thus resort to game theory models. Further, in [37] we tackle the problem of spectrum and infrastructure sharing addressed here by a noncooperative approach and formalize it as a generalized Nash equilibrium problem, where the operators strategies consist of the choice of coalition and the fraction of coalition cost to pay. However, the non-cooperative approach limits the stability analysis to the action of the single player, while the cooperative approach allows to determine whether a coalition is stable or not also in terms of joint actions of its members.

\section{The PROBLEM}

\section{A. Problem definition}

We consider a set $O$ of MNOs which provide data services to users of a dense urban area through pre-4G macrocell networks but have plans to upgrade their RAN technology by deploying $4 \mathrm{G}$ small cells. We assume MNOs inherit the user share from their individual current networks: being $N$ the number of users that populates the given area, each MNO $i \in O$ has a fixed market share $\sigma_{i}$, that is, user churning is assumed to be null. We also assume that at least one MNO owns a spectrum license of $b_{i}$ units of bandwidth ${ }^{5}$ which it plans to put to use for the network of small cells. Each MNO may decide to deploy its individual network of small cells or collaborate with other MNOs to deploy a shared one. When a

\footnotetext{
${ }^{5}$ MNOs with no spectrum license are represented by $b_{i}=0$.
}

set of MNOs decides to deploy a shared network, we assume they will agree on aggregating their individual spectrum. Let $\mathcal{S}$ be the set of all possible coalitions that can be created, that is, the set of all the possible subsets of MNOs agreeing to deploy a shared network. If coalition $s \in \mathcal{S}$ is created, it will deploy a shared network infrastructure of total cost $\widetilde{c}_{s}$ which has to be divided among its member MNOs. Applying a simple data pricing model, each MNO $i$ incurs revenues $\widetilde{r}_{s}^{i}$ from its user subscriptions when in $s$. The case when MNOs in $s$ agree to share the coalition cost $\widetilde{c}_{s}$ but keep their individual revenues $\widetilde{r}_{s}^{i}$ is formalized as a cooperative game without transferable payoffs. Alternatively, the case when MNOs would be willing to give away also part of their revenues is modeled as a cooperative game with transferable payoffs. The core and nucleolus solution concepts are then leveraged to determine stable cost divisions.

\section{B. Cost and revenues definition}

Since this work addresses sharing at the RAN, the adopted cost model accounts only for radio equipments cost and a simplified leased line pricing model for the backhaul transmission cost as in [38]. Moreover, in [39], it is argued that the cost associated with the RAN dominates the remaining cellular network cost.

An investment period of duration $D$ (months) has been considered. Let $g_{s}$ be the total cost incurred in $D$ by coalition $s$ from activating and operating one small cell BS: $g_{s}$ accounts for the capital (CAPEX) and operational (OPEX) expenditures of the radio equipment, the backhaul transmission cost and the site build-out cost. We denote by $\widetilde{b}_{s}$ the aggregated spectrum of coalition $s$, that is, $\widetilde{b}_{s}=\sum_{i \in s} b_{i}$, whereas by $\beta_{s}$ the number of MNOs in $s$ which own a spectrum license, that is, $\beta_{s}=\left|\left\{i \in s: b_{i}>0\right\}\right|$. Let $g_{\text {small }}^{\mathrm{c}, \mathrm{r}}$ be the radio equipment CAPEX of a typical small cell BS supporting a single carrier. Given that a small cell BS activated by coalition $s$ aggregates $\beta_{s}$ carriers, it has to support $\beta_{s}-1$ additional carriers. As in [40], we consider a fixed cost for each additional carrier, calculated as a percentage $\phi$ of the cost $g_{\text {macro }}^{\mathrm{c}, \mathrm{r}}$ of a singlecarrier macrocell BS. The total radio equipment CAPEX of a small cell BS activated by coalition $s, g_{s}^{\mathrm{c}, \mathrm{r}}$, is then given as follows:

$$
g_{s}^{\mathrm{c}, \mathrm{r}}=g_{\text {small }}^{\mathrm{c}, \mathrm{r}}+\left(\beta_{s}-1\right) \phi g_{\text {macro }}^{\mathrm{c}, \mathrm{r}}
$$

The Operations and Maintenance (O\&M) annual cost of the radio equipment is calculated as a percentage $\xi$ of the corresponding total radio CAPEX $g_{s}^{c, r}[38,41]$. The considered backhaul leased line pricing model consists of an upfront fee $g^{\mathrm{c}, \mathrm{b}}$ and the annual leasing cost $g_{s}^{\mathrm{o}, \mathrm{b}}$ which are incurred for each BS activated by coalition $s$. We assume that, in the worst case, $g_{s}^{\mathrm{o}, \mathrm{b}}$ is proportional to the total amount of spectrum (bandwidth) aggregated by any of the BSs of coalition $s\left(\widetilde{b}_{s}\right)$. Let $g_{0}^{\mathrm{o}, \mathrm{b}}$ be the annual leased line cost for a reference carrier of $b_{0}$ units of bandwidth. We then set $g_{s}^{\mathrm{o}, \mathrm{b}}$ equal to $\widetilde{b}_{s} g_{0}^{\mathrm{o}, \mathrm{b}} / b_{0}$. Let $g^{c, s}$ denote the site build-out cost. Finally, the total cost 
$g_{s}$ incurred by coalition $s$ from a single small cell BS in $D$ is given by:

$$
g_{s}=g_{s}^{\mathrm{c}, \mathrm{r}}+g^{\mathrm{c}, \mathrm{b}}+g^{\mathrm{c}, \mathrm{s}}+\frac{D}{12}\left(\xi g_{s}^{\mathrm{c}, \mathrm{r}}+g_{s}^{\mathrm{o}, \mathrm{b}}\right) .
$$

The considered cost parameter values (Table I) refer to HSPA technology as in $[38,40]$, given that, to the best of our knowledge, CA-enabled equipment cost are not made publicly available by any vendor. Such cost should nevertheless represent a good estimate, at least in orders of magnitude, since as argued by Johansson et al. [40], the physical infrastructure cost of new radio access technologies tend to be similar to the previous ones.

\begin{tabular}{lll}
\hline Symbol & \multicolumn{1}{c}{ Description } & Value \\
\hline$g_{\text {small }}^{\text {c,r }}$ & Single-carrier small cell BS radio equipment cost & $3000 €[38]$ \\
$g_{\text {macro }}$ & Single-carrier macro cell BS radio equipment cost & $20000 €[38]$ \\
$\phi$ & Cost coefficient per additional carrier & $0.017[40]$ \\
$g^{c, b}$ & Upfront fee for backhaul & $2000 €[38]$ \\
$b_{0}$ & Bandwidth of the reference carrier & $5 \mathrm{MHz}[38]$ \\
$g_{0}^{\text {o,b }}$ & Annual leased line cost of the reference carrier & $2000 €[38]$ \\
$g^{\mathrm{c}, \mathrm{s}}$ & Site buildout cost & $2000 €[38]$ \\
$\xi$ & O\&M annual percentage & $15 \%[41]$ \\
\hline
\end{tabular}

TABLE I: BS cost model parameters

As in [36] and [37], the revenues $\widetilde{r}_{s}^{i}$ incurred by MNO $i$ in coalition $s$ are calculated according to a simple data service pricing model, where the latter is defined in terms of the average data rate perceived by users of $s$. Let $\rho_{s}^{n o m}\left(u_{s}\right)$ be the nominal rate coalition $s$ can provide to its users by activating $u_{s}$ BSs. For a given level of Signal to Interference and Noise Ratio (SINR) and a given system bandwidth, the nominal user rate in LTE is the maximum rate perceived by a single user when assigned all downlink LTE resource blocks from its serving BS. The downlink SINR is a function of the number of BSs activated by the coalition the user belongs to: a larger number of BSs results in the user being on the average closer to its serving BS, and therefore receiving a stronger signal, but also closer to the interfering ones ${ }^{6}$. The average rate $\rho_{s}\left(u_{s}\right)$ perceived by a user in coalition $s$ can be defined in terms of $\rho_{s}^{n o m}\left(u_{s}\right)$ and of the load of its serving BS:

$$
\rho_{s}\left(u_{s}\right)=\rho_{s}^{n o m}\left(u_{s}\right)(1-\eta)^{\frac{\sum_{i \in s} \sigma_{i} N}{u_{s}}}, \quad \forall s \in \mathcal{S},
$$

where parameter $\eta$ is the user activity factor representing the probability that a user is actually active in his/her serving BS, $\sum_{i \in s} \sigma_{i} N$ is the total number of users of coalition $s$ whereas $\left(\sum_{i \in S} \sigma_{i} N\right) / u_{s}$ gives the average number of users served by one BS. To obtain $\rho_{s}\left(u_{s}\right)$, the nominal rate is then scaled down by the factor $(1-\eta)\left(\sum_{i \in s} \sigma_{i} N\right) / u_{s}$ representing the average congestion level at a serving BS.

Let $\delta$ denote the monthly price per user and per unit (Mbps) of data service. As $\rho_{s}\left(u_{s}\right)$ represents the average user rate provided by coalition $s \in \mathcal{S}$ when it activates $u_{s} \mathrm{BSs}^{7}$, the

\footnotetext{
${ }^{6}$ When calculating the nominal user rate, any other BS transmission will use at least a subset of the available resource blocks and therefore unavoidably interfere.

${ }^{7}$ The simulation set up to obtain $\rho_{s}\left(u_{S}\right)$ as a function of $u_{S}$ for each $s \in \mathcal{S}$ is explained in details in Section V-A.
}

revenues $r_{s}^{i}$ each member MNO $i \in s$ can incur when in $s$ at the end of the investment lifetime $D$, are then modeled linearly in $\rho_{s}\left(u_{s}\right)$ :

$$
r_{s}^{i}=\delta D \sigma_{i} N \rho_{s}\left(u_{s}\right), \quad \forall i \in s .
$$

Let $\widetilde{u}_{s}$ be the number of BSs that maximizes the global return on investment of coalition $s$ calculated as:

$$
\widetilde{u}_{s}=\underset{\substack{u_{s} \in \mathbb{Z}_{+} \\ u_{s} \leq U_{\max }}}{\operatorname{argmax}}\left(\sum_{i \in s} \delta D \sigma_{i} N \rho_{s}\left(u_{s}\right)-g_{s} u_{s}\right), \quad \forall s \in \mathcal{S},
$$

where $U_{\max }$ is the maximum number of small cell sites coalition $s$ can activate in the area.

Finally, the revenues $\widetilde{r}_{s}^{i}$ of MNO $i$ from coalition $s$ and the total cost $\widetilde{c_{s}}$ of coalition $s$ are the following:

$$
\begin{array}{cl}
\widetilde{r}_{s}^{i}=\delta D \sigma_{i} N \rho_{s}\left(\widetilde{u}_{s}\right), & \forall s \in \mathcal{S}, \forall i \in s, \\
\widetilde{c}_{s}=g_{s} \widetilde{u}_{s}, & \forall s \in \mathcal{S} .
\end{array}
$$

\section{Cooperative game models}

In this section we describe the two cooperative game theory models we developed for the problem. The first one is a Non Transferable Utility (NTU) game, namely we assume that players share the network infrastructure cost, but each keeps its own revenue (Section IV-A), while the second one is a Transferable Utility (TU) game, namely, beside sharing the cost, we allow players to partially transfer their revenue to others (Section IV-B).

The NTU model represents indeed a more intuitive scenario as MNOs incur revenues from their individual share of users. However, we define the player payoffs in terms of their profits, i.e., as revenues minus cost; therefore, if an MNO benefits from being in a coalition, e.g., due its aggregated spectrum resources, and has no incentive to leave the coalition, even when giving away part of its revenue to the others, then this is worth being investigated by means of the TU model. In other words, the TU model allows to analyze at what extent a coalition is valuable to the MNOs. ${ }^{8}$

For both games, we want to determine whether the grand coalition is selected and, if so, how to make it stable. Thus, we look for the elements of the core, namely the payoff allocations which guarantee that there is no incentive neither for an MNO to leave the grand coalition and build a network by itself nor for any subset of MNOs to create their own coalition/shared network. In other words, whenever the core is nonempty, the grand coalition is preferred by all MNOs.

\section{A. A Non Transferable Utility game model}

We model the problem as a NTU cooperative game $(O, V)$, where the set of players coincides with the set $O$ of MNOs. The set-valued mapping $V$ assigns a set of feasible payoff vectors $V(s)$ to each coalition $s \in \mathcal{S}$ and is defined as follows:

$$
V(s)=\left\{\left(p_{i}\right)_{i \in O}: \sum_{i \in s} p_{i} \leq \sum_{i \in s} \widetilde{r}_{s}^{i}-\widetilde{c}_{s}, \quad p_{i} \leq \widetilde{r}_{s}^{i}, \quad \forall i \in s\right\} .
$$

\footnotetext{
${ }^{8}$ See Section VI for numerical examples.
} 
The value of the payoffs $p_{i}$ is bounded by the inequalities described above. Inequality $\sum_{i \in s} p_{i} \leq \sum_{i \in S} \widetilde{r}_{s}^{i}-\widetilde{c}_{s}$ guarantees that the sum of the payoffs of the players does not exceed the overall payoff, which is given by the difference between the sum of the revenues and the coalitional cost. Inequalities $p_{i} \leq \widetilde{r}_{s}^{i}$ make sure that the revenues are not transferred among players by limiting each player's payoff to its respective revenue.

We aim at determining whether the grand coalition is selected by the players or not, and how they decide to share the network cost among them. Thus, we study the core of the game, namely the set of payoff vectors that make the grand coalition preferable to any sub-coalition. To formally define the core, the Pareto efficient frontier $F$ of the set $V(O)$ must be defined as follows:

$F=\left\{\left(p_{i}\right)_{i \in O}: \sum_{i \in O} p_{i}=\sum_{i \in O} \widetilde{r}_{O}^{i}-\widetilde{c}_{O}, \quad p_{i} \leq \widetilde{r}_{O}^{i}, \quad \forall i \in O\right\}$.

The core of the game is then defined as

$$
C=F \backslash \bigcup_{s \in \mathcal{S}} \text { int } V(s),
$$

where \ denotes the difference between two sets and int denotes the interior of a set.

\section{B. A transferable utility game model}

If we assume that players may partially transfer their revenue to others, then the corresponding model is a Transferable Utility game $(O, v)$, where $O$ is the set of players and $v$ is the characteristic function, i.e., a real-valued function which assigns to each coalition $s \in \mathcal{S}$ its overall payoff defined as

$$
v(s)=\sum_{i \in s} \widetilde{r}_{s}^{i}-\widetilde{c}_{s} .
$$

Notice that this TU game is equivalent to the NTU game described in Section IV-A where the constraints $p_{i} \leq \widetilde{r}_{s}^{i}$ for any $i \in s$ are removed from the definition of $V(s)$.

Similarly to the NTU model, we are interested in finding the set of payoff vectors that make the grand coalition preferable to any sub-coalition, that is the core of the TU game, which is defined as

$C=\left\{\left(p_{i}\right)_{i \in O}: \sum_{i \in O} p_{i}=v(O), \quad \sum_{i \in s} p_{i} \geq v(s), \quad \forall s \subset O\right\}$.

We remark that we have defined the characteristic function $v$ assuming the joint strategy space of coalition $s$ is the number of BSs it activates $\left(0 \leq u_{s} \leq U_{\max }\right)$. Further, the utility of $s$ from activating $u_{s}$ BSs is given by the corresponding global return on investment, $\sum_{i \in s} r_{s}^{i}\left(u_{s}\right)-c_{s}\left(u_{s}\right)$, where such utility depends only on $u_{s}$ and it is not affected by the actions of $i \notin s$. Therefore, $\widetilde{u}_{s}$ represents the strategy of coalition $s$ whereas $v(s)=\sum_{i \in s} \widetilde{r}_{s}^{i}-\widetilde{c}_{s}$ its overall payoff. Although the overall payoff of a coalition is determined maximizing its total return on investment, which does not necessarily maximize the individual return on investment of each of its member MNOs, when such payoff is distributed among them according to a solution in the core, no MNO has an incentive to deviate.

\section{A two MNOs example}

Assume that there are only two MNOs, i.e., $O=\{A, B\}$. For the NTU game (see Figure 1a), the set of feasible payoff vectors corresponding to the grand coalition $\{A, B\}$ is

$V_{N T U}(\{A, B\})=\left\{\begin{aligned} p_{A} & +p_{B} \leq \widetilde{r}_{\{A, B\}}^{A}+\widetilde{r}_{\{A, B\}}^{B}-\widetilde{c}_{\{A, B\}} \\ \left(p_{A}, p_{B}\right): & p_{A} \leq \widetilde{r}_{\{A, B\}}^{A} \\ p_{B} & \leq \widetilde{r}_{\{A, B\}}^{B}\end{aligned}\right\}$.

The Pareto efficient frontier $F_{N T U}$ of the set $V_{N T U}(\{A, B\})$ is the line segment with extreme points

$$
\begin{aligned}
& \Pi_{A}=\left(\begin{array}{ll}
\widetilde{r}_{\{A, B\}}^{A}, & \widetilde{r}_{\{A, B\}}^{B}-\widetilde{c}_{\{A, B\}}
\end{array}\right), \\
& \Pi_{B}=\left(\begin{array}{ll}
\widetilde{r}_{\{A, B\}}^{A}-\widetilde{c}_{\{A, B\}}, & \widetilde{r}_{\{A, B\}}^{B}
\end{array}\right),
\end{aligned}
$$

where in $\Pi_{A}$ the coalitional cost $\widetilde{c}_{\{A, B\}}$ is entirely paid by MNO $B$, while in $\Pi_{B}$ it is entirely paid by $A$. Therefore, the core $C_{N T U}$ (the bold segment in the figure) is obtained as the difference between $F_{N T U}$ and the union of the interiors of the two halfplanes representing the feasible payoffs for the single player coalitions:

$$
\begin{aligned}
& \text { int } V(\{A\})=\left\{\left(p_{A}, p_{B}\right): p_{A}<v(\{A\})\right\} \text {, } \\
& \text { int } V(\{B\})=\left\{\left(p_{A}, p_{B}\right): p_{B}<v(\{B\})\right\} \text {, }
\end{aligned}
$$

where $v(\{A\})=\widetilde{r}_{\{A\}}^{A}-\widetilde{c}_{\{A\}}$ and $v(\{B\})=\widetilde{r}_{\{B\}}^{B}-\widetilde{c}_{\{B\}}$.

In fact, such halfplanes represents the sets of vectors of payoff such that the single player would earn more alone than joining the grand coalition. Therefore, we can write the core $C_{N T U}$ as follows:

$C_{N T U}=\left\{\begin{array}{ll} & p_{A}+p_{B}=\widetilde{r}_{\{A, B\}}^{A}+\widetilde{r}_{\{A, B\}}^{B}-\widetilde{c}_{\{A, B\}} \\ \left(p_{A}, p_{B}\right): & v(\{A\}) \leq p_{A} \leq \widetilde{r}_{\{A, B\}}^{A} \\ & v(\{B\}) \leq p_{B} \leq \widetilde{r}_{\{A, B\}}^{B}\end{array}\right\}$.

Instead, for the TU game (see Figure 1b), the set of feasible payoff vector is the halfplane

$V_{T U}(\{A, B\})=\left\{\left(p_{A}, p_{B}\right): p_{A}+p_{B} \leq \widetilde{r}_{\{A, B\}}^{A}+\widetilde{r}_{\{A, B\}}^{B}-\widetilde{c}_{\{A, B\}}\right\}$,

the Pareto efficient frontier is the line

$$
F_{T U}=\left\{\left(p_{A}, p_{B}\right): p_{A}+p_{B}=\widetilde{r}_{\{A, B\}}^{A}+\widetilde{r}_{\{A, B\}}^{B}-\widetilde{c}_{\{A, B\}}\right\},
$$

and the core

$$
C_{T U}=\left\{\begin{array}{ll} 
& p_{A}+p_{B}=\widetilde{r}_{\{A, B\}}^{A}+\widetilde{r}_{\{A, B\}}^{B}-\widetilde{c}_{\{A, B\}} \\
\left(p_{A}, p_{B}\right): & p_{A} \geq v(\{A\}) \\
& p_{B} \geq v(\{B\})
\end{array}\right\} .
$$

Notice that the core of the NTU game is a subset of the TU one. In the example depicted in Figure 1, the grand coalition provides MNO $B$ with a strictly positive margin with respect to investing alone, even if it pays the entire $\widetilde{c}_{\{A, B\}}$ cost, that is, $\widetilde{r}_{\{A, B\}}^{B}-\widetilde{c}_{\{A, B\}}-v(\{B\})>0$. Therefore, it is still profitable for $B$ to be in the grand coalition even it transfers part of its revenues to $A$ (represented by payoff vectors in $C_{T U} \backslash C_{N T U}$ ).

The following relations between the parameter values determine whether the core of each two players game is empty or not: 


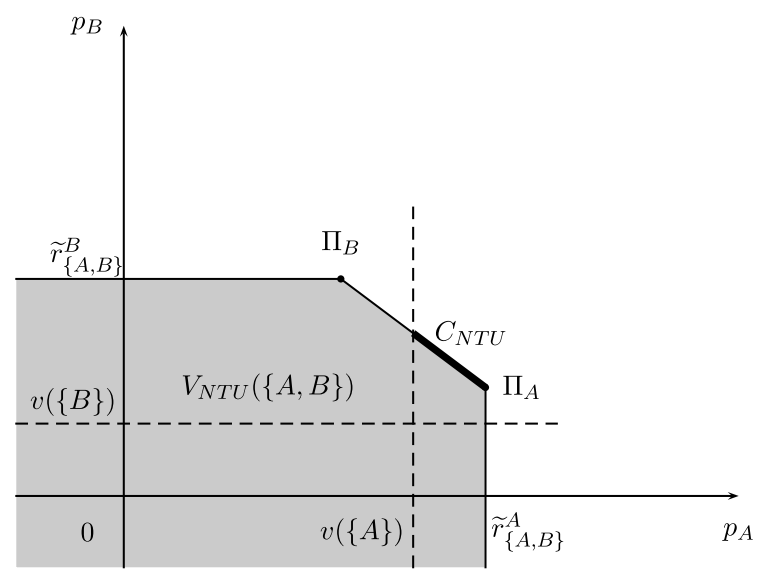

(a) NTU game

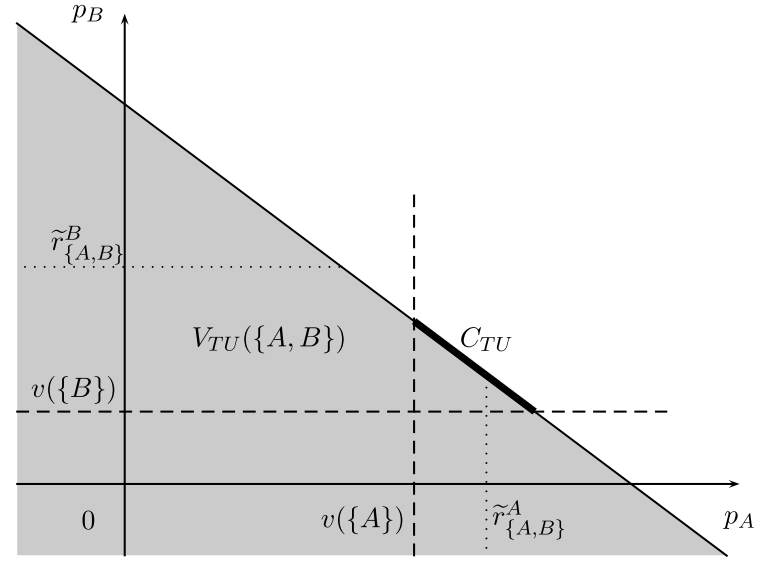

(b) TU game

Fig. 1: A two-players example: Pareto frontier and core

1) If $v(\{A, B\})<v(\{A\})+v(\{B\})$, that is,

$\widetilde{c}_{\{A, B\}}-\left(\widetilde{c}_{\{A\}}+\widetilde{c}_{\{B\}}\right)>\left(\widetilde{r}_{\{A, B\}}^{A}+\widetilde{r}_{\{A, B\}}^{B}\right)-\left(\widetilde{r}_{\{A\}}^{A}+\widetilde{r}_{\{B\}}^{B}\right)$,

then the core of both games is empty as both MNOs are better off investing alone. Roughly speaking, if the additional revenues generated from the grand coalition do not cover its additional cost, than the grand coalition is not stable.

2) Otherwise, if $v(\{A, B\}) \geq(v\{A\})+v(\{B\})$, then the core of the TU game is nonempty. In particular, when $v(\{A, B\})=v(\{A\})+v(\{B\})$, it consists of a single payoff vector, $\left(p_{A}=v_{\{A\}}, p_{B}=v_{\{B\}}\right)$, which corresponds to the case when MNOs are indifferent between cooperating or not. As for the NTU game:

2a) If either $v(\{A\})>\widetilde{r}_{\{A, B\}}^{A}$ or $v(\{B\})>\widetilde{r}_{\{A, B\}}^{B}$, then the core of the NTU game is empty. Notice that $v(\{A\})>$ $\widetilde{r}_{A B}^{A}$ means MNO $A$ is better off alone, even if $B$ could pay for the entire $\widetilde{c}_{\{A, B\}}$ cost.

2b) Otherwise, if $v(\{A\}) \leq \widetilde{r}_{\{A, B\}}^{A}$ and $v(\{B\}) \leq \widetilde{r}_{\{A, B\}}^{B}$, then also the core of the NTU game is nonempty.

\section{Computational tests}

\section{A. Simulation environment}

As in [36] and [37], a simulation environment has been set up in Matlab to obtain the average user rate $\rho_{s}\left(u_{s}\right)$ for each coalition $s$ as function of the number $u_{s}$ of activated small cell BSs varying from 1 to $U_{\max }$. The $u_{s}$ BSs and 10 sample users are uniformly distributed in a pseudo-random fashion on the considered square area. The downlink SINR for a reference system bandwidth of a sample user of coalition $s$, when $s$ activates $u_{s} \mathrm{BSs}$, is given by:

$$
\operatorname{SINR}_{s}=\frac{P_{i}}{l_{s}\left(\sum_{\substack{1 \leq j \leq u_{s} \\ j \neq i}} P_{j}\right)+P_{\text {noise }}}, \quad \forall s \in \mathcal{S},
$$

where $P_{i}$ is the signal power the sample user receives from its serving BS, whereas $\sum_{1 \leq j \leq u_{s}, j \neq i} P_{j}$ is the power received from interfering (non-serving) ones. The received signal power is calculated according to the following three-parameter path loss model (transmitted signal power $P_{t x}$, fixed path loss $C_{p l}$ and path loss exponent $\Gamma$ ), defined within the GreenTouch Consortium [42]:

$$
P_{r x}[d B m]=P_{t x}[d B m]-C_{p l}[d B]-10 \Gamma \log (d[k m]),
$$

where $d$ is the sample user-BS distance. The captured interference is then scaled down by the load of coalition $s$, $l_{s}=1-(1-\eta)^{\frac{\sum_{i \in s} \sigma_{i} N}{u_{s}}}$, as users are characterized by an activity factor $\eta$. $P_{\text {noise }}$ is the white gaussian noise power for the reference system bandwidth.

The resulting SINR is then mapped to LTE spectral efficiency according to a multilevel SINR-to-spectral efficiency scheme [42]. Multiplying the obtained spectral efficiency by the coalition aggregated bandwidth $\widetilde{b}_{s}$, we obtain the nominal user rate $\rho_{s}^{\text {nom }}\left(u_{s}\right) .100$ simulation iterations are run for each value of $u_{s}$ so that an average value for $\rho_{s}^{n o m}\left(u_{s}\right)$ is obtained across all sample users and iterations. Finally, $\rho_{s}\left(u_{s}\right)$ is obtained from $\rho_{s}^{\text {nom }}\left(u_{s}\right)$ as defined in (3).

\section{B. Instances}

We consider instances with $3 \mathrm{MNOs}^{9}$, namely $A, B$ and $C$ and a $4 \mathrm{~km}^{2}$ area populated by 20000 users. $U_{\max }$ is set to 10000 , which is an arbitrarily large number of small cells for the considered area size; however, the number of activated small cells by any coalition does not exceed 1500 for all the considered instances. Parameter $\delta$, which represents the monthly price per unit of service and per user, is set equal to equidistant values obtained discretizing the range $[0.5,3]$ with a 0.01 step. We set up 5 scenarios (S1-S5) with different mixtures of market shares and "spectrum shares"10 as shown in Table II. The values of the bandwidth associated with the spectrum license of each MNO $b_{i}$, are set to standardized

\footnotetext{
${ }^{9}$ The considered number of MNOs is common for most countries, as far as facility-based operators are concerned [43]. [25] and [34] also consider 3 MNOs. Nevertheless, the proposed approach can be easily extended to more MNOs.

${ }^{10}$ The term "spectrum share" is used analogously with market share to represent the weight of the spectrum of an MNO w.r.t. to the total obtained aggregating the spectrum of all MNOs $\left(b_{i} / \sum_{j \in O} b_{j}\right)$.
} 
bandwidths for LTE/LTE-A $(\{1.4,3,5,10,15,20\} \mathrm{MHz})$ [13]. In particular, scenarios S4 and S5 aim at representing cases that may arise under traditional and recent design of spectrum auctions. The extreme case in which only one MNO in the area has succeeded to obtain a spectrum license from the latest auction has been considered; we assume such MNO is either the smallest MNO (S4), for instance, a new entrant which has benefited from a set-aside spectrum policy ${ }^{11}$ [3], or the incumbent (S5), which is the most likely to be the highest bidder in a traditional auction.

\begin{tabular}{|c|c|c|c|c|c|c|c|}
\hline \multirow{2}{*}{\multicolumn{3}{|c|}{\begin{tabular}{|lll}
\multicolumn{2}{|c|}{$\mathbf{S 1}$} \\
$A$ & $B$ & $C$
\end{tabular}}} & \multicolumn{2}{|r|}{ S2 } & S3 & S4 & S5 \\
\hline & & & $\bar{A}$ & $B C$ & $\begin{array}{lll}A & B & C\end{array}$ & $\begin{array}{lll}A & B & C\end{array}$ & $\begin{array}{lll}A & B & C\end{array}$ \\
\hline$u_{l}$ & $1 / 31 / 3$ & $1 / 3$ & $1 / 3$ & $31 / 31 / 3$ & $\begin{array}{|lll|}0.1 & 0.3 & 0.6 \\
\end{array}$ & $\begin{array}{llll}0.1 & 0.3 & 0.6\end{array}$ & 0.10 .30 \\
\hline$b_{i}$ & $\begin{array}{ll}5 & 5 \\
\end{array}$ & 5 & 1.4 & 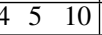 & $\begin{array}{|lll|}5 & 5 & 5 \\
\end{array}$ & $\begin{array}{|lll|}15 & 0 & 0 \\
\end{array}$ & $\begin{array}{ll}0 & 15 \\
\end{array}$ \\
\hline
\end{tabular}

TABLE II: Scenarios

Parameters notation and their corresponding values are summarized in Table III.

\begin{tabular}{clc}
\hline Symbol & \multicolumn{1}{c}{ Description } & Value \\
\hline$O$ & Set of MNOs & $\{A, B, C\}$ \\
$\mathcal{S}$ & Set of nonempty coalitions & $2 O \backslash \emptyset$ \\
$N$ & Total number of users in the area & 20000 \\
$A$ & Area size & $4 \mathrm{~km}^{2}$ \\
$U_{\max }$ & Max. number of BSs in the area & 10000 \\
$\delta$ & Monthly price of 1 Mbps & {$[0.5,3] € / \mathrm{Mbps}$} \\
$D$ & Investment lifetime & 120 months $[45]$ \\
$\eta$ & User activity factor & 0.01 \\
\hline
\end{tabular}

TABLE III: Sets, parameters and corresponding values

\section{NUMERICAL RESULTS ANALYSIS}

In this section we discuss the results obtained applying first the NTU game model and then the TU game model. Our goal is to highlight the impact of the three main parameters of the problem, namely $\delta$, market share and spectrum share, on the existence of the core and on its features and on the nucleolus. The nucleolus is a well known solution of NTU and TU games (see, e.g., [46,47]). We use the nucleolus as a suggested solution, as it always belongs to the core, if the core is nonempty, and therefore represents a stable way of assigning payoffs to players. Roughly speaking, the nucleolus minimizes the largest dissatisfaction of the coalitions, thus reducing the inequity among the coalitions, where the dissatisfaction is related to the difference between the coalition value and what its members receive according to the nucleolus.

Across all considered scenarios, when $\delta \in[0.5,0.53]$, neither the grand coalition nor subcoalitions find it profitable to invest, thus the core is trivial as it collapses to only one point corresponding to zero investment and thus zero revenues.

\footnotetext{
${ }^{11}$ Despite some countries regulator efforts to encourage competition in mobile networks, by introducing spectrum set-asides during auctions and relaxing their coverage requirements, new entrants do not always succeed in deploying a network which may lead to inefficient spectrum allocations or eventually with the set-aside spectrum ending up in the hands of incumbent MNOs [44].
}

Instead, for $\delta \in[0.58,3]$, the core of both games is nonempty (see Table IV).

Therefore in Sections VI-A and VI-B, we focus our analysis on instances with a nonempty core. For such instances, as payoff allocations in the core make the grand coalition preferable to any subcoalition, only the grand coalition is analyzed. Instead, for the few particular instances with an empty core, we investigate subcoalitions (Section VI-C).

In Section VI-D, we assess the MNOs' gain from sharing with respect to building individual networks.

Figures 2 and 3 report the core of the NTU and the TU game, respectively, for $\delta \in\{0.75,1.5,3\}$ for each considered scenario (S1-S5).

As we are interested in how the players share the network infrastructure cost, we introduce three values $\alpha_{A}, \alpha_{B}$ and $\alpha_{C}$ which represent the fraction of the overall cost paid by player $A, B$ and $C$, respectively. The payoff of a player $i$ in the grand coalition $O$ can be therefore written as

$$
p_{i}=\widetilde{r}_{O}^{i}-\alpha_{i} \vec{c}_{O}^{i} .
$$

The core is represented in the $\left(\alpha_{A}, \alpha_{B}\right)$ plane, as $\alpha_{C}=1-$ $\alpha_{A}-\alpha_{B}$.

As for the NTU game, in each sub-figure of Figure 2 the Pareto efficient frontier $F$ is represented by the triangle with vertices $(0,0)(0,1)(1,0)$, where the diagonal line connecting $(0,1)$ and $(1,0)$ represents the payoff values such that $\alpha_{C}=0$. The light grey areas represent the sets $V(s)$ for all the subcoalitions. The core is thus represented by the dark grey area. Beside the core, the nucleolus is reported with a white circle and the market share with a black triangle.

As for the TU game, in each sub-figure of Figure 3 the core is represented by the dark grey area, while dashed lines represent lines $\alpha_{A}=0, \alpha_{B}=0$ and $\alpha_{C}=0$. The color and symbolic code of Figure 3 are the same as the one of Figure 2.

\section{A. NTU game results}

1) Impact of $\delta$ : For all the considered scenarios, the core size enlarges with the increasing value of $\delta$, namely the range of the acceptable values of $\alpha_{A}, \alpha_{B}$ and $\alpha_{C}$ increases. Let us consider for instance scenario $\mathbf{S 1}$ (Figure 2a): for $\delta=0.75, \alpha_{A}$ ranges from about 0.25 to about 0.42 , while for $\delta=3$ it may rise up to about 0.8 . Roughly speaking, with the increasing value of $\delta$ and therefore the increasing revenues, players accept more ways of dividing the costs and accept to bear a higher fraction of costs. Further, they may also accept to free one of the players of its fraction of costs. In scenario $\mathbf{S 1}$ each player can be freed from the network cost, as $\alpha_{A}, \alpha_{B}$ and $\alpha_{C}$ can all be equal to 0 for $\delta=3$, due to the symmetry of the core. However, only one player at a time can be freed from the cost, the other two agreeing to share the overall amount. In fact, in scenario $\mathbf{S 1}$ the market shares as well as the spectrum shares are equal: this results in a symmetric core and makes the market share coincide with the nucleolus. Market share and spectrum share have an impact of the shape of the core, as it will be discussed in the following paragraphs. 

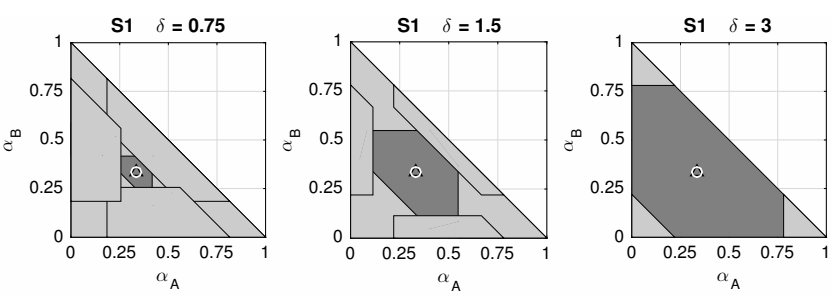

(a) Scenario $\mathrm{S} 1$
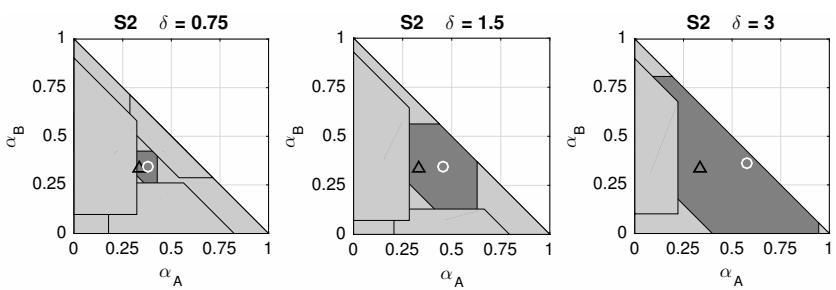

(b) Scenario S2
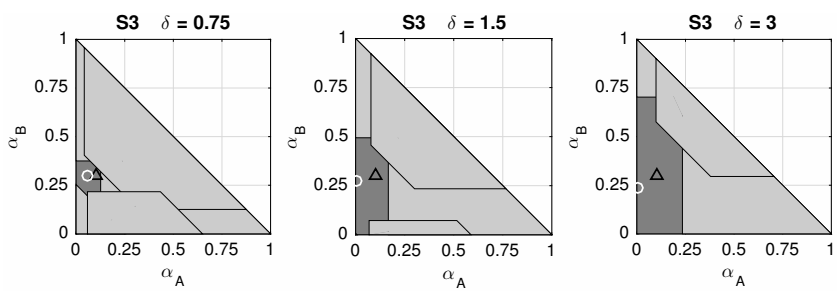

(c) Scenario S3
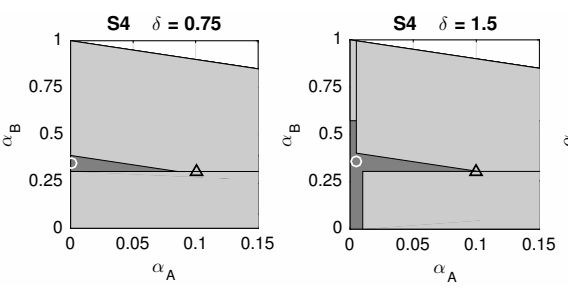

(d) Scenario S4
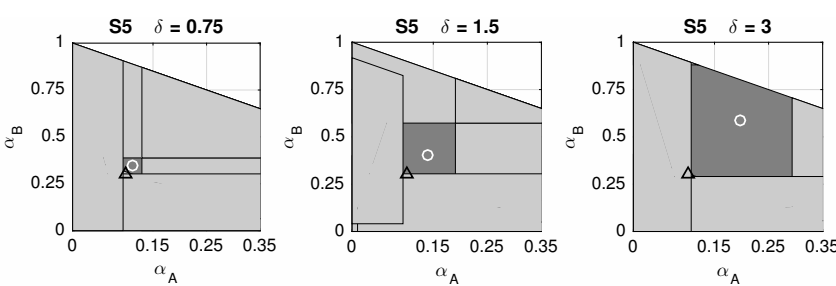

(e) Scenario S5

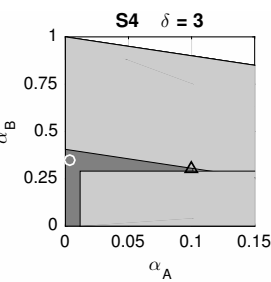

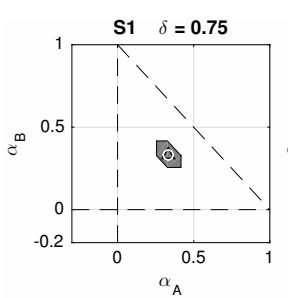
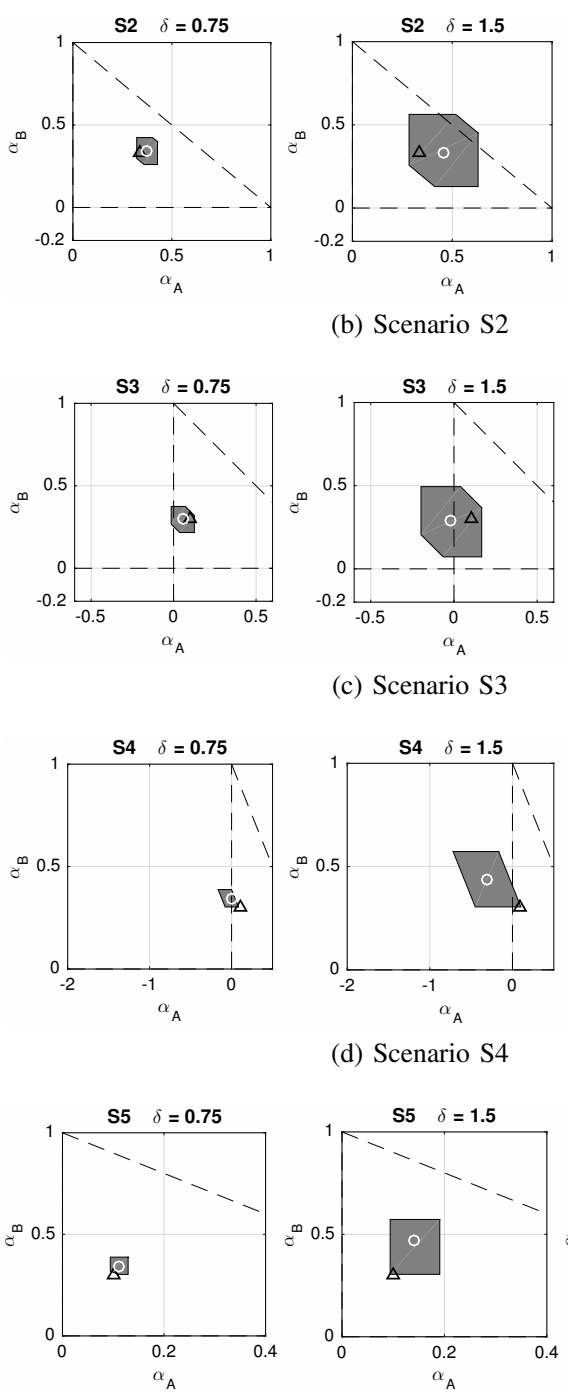

(e) Scenario S5

(a) Scenario $\mathrm{S} 1$

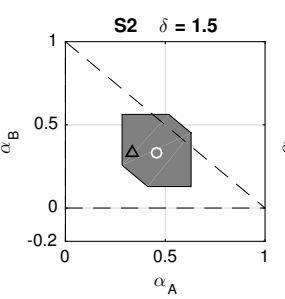

(b) Scenario S2

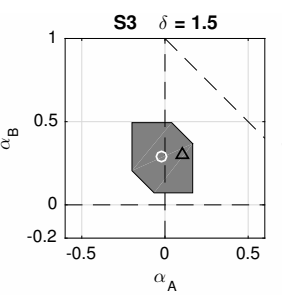

(c) Scenario $\mathrm{S} 3$

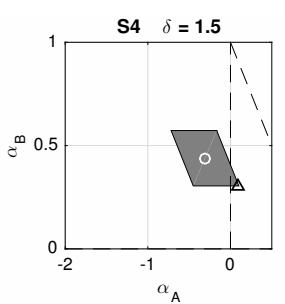

(d) Scenario $\mathrm{S} 4$
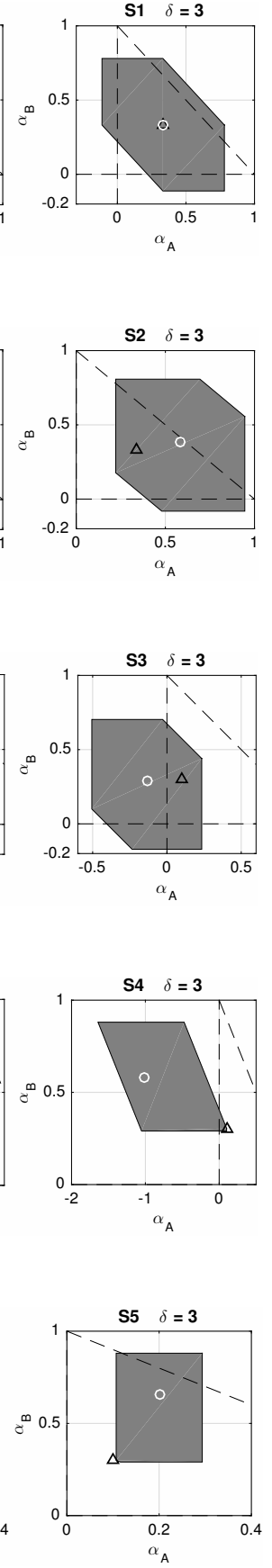

Fig. 2: NTU game results: core, nucleolus and market shares

2) Impact of the spectrum share: To highlight the impact of the spectrum share let us compare scenarios S1 (Figure 2a), where all the players have the same market and spectrum share, and S2 (Figure 2b), where all the players have the same market share, but different spectrum shares (1.4:5:10 $\mathbf{M H z}$, respectively). For scenario $\mathbf{S 2}$ the core is not symmetric, differently from scenario $\mathbf{S} 1$. The acceptable fraction is somehow inversely dependent from the spectrum share: the highest the spectrum share the smallest the minimum fraction allowed. Thus, for $\delta \geq 1.5, \alpha_{C}$ can be equal to 0 , as $A$ and $B$ are willing to share the overall cost in order to exploit the spectrum provided by $C$. For the highest value of $\delta, \alpha_{A}$ can reach 0.95 , while $\alpha_{B}$ is at most 0.8 . The market share belongs to the core but it never coincides with the nucleolus, which gets closer to the line representing $\alpha_{C}=0$ as $\delta$ increases.

3) Impact of the market share: To highlight the impact of the market shares, let us compare scenarios S1 (Figure 2a), where all the players have the same market share, and $\mathbf{S 3}$ (Figure 2c), where all the players have the same spectrum share, but different market shares (0.1:0.3:0.6, respectively). In scenario $\mathbf{S 3}$ the core is not symmetric, although as for scenario S1 it enlarges with the increasing values of $\delta$. The range of acceptable values of $\alpha_{B}$ is greater than the range of acceptable
Fig. 3: TU game results: core, nucleolus and market shares 
values of $\alpha_{A}$. It is somehow proportional to the market share: in fact $B$ has three times the users of $A$ and for $\delta=3 \alpha_{A} \in$ $[0,0.25]$ while $\alpha_{B} \in[0,0.75]$. Player $C$, which has the highest number of users, may accept to pay the overall BSs cost so as to profit of the other two's spectrum: in fact the point $\alpha_{A}=$ $\alpha_{B}=0$ is in the core. Although the market share is in the core, it does not coincide with the nucleolus, not even for $\delta=0.75$. For higher values of $\delta$ the nucleolus suggests to keep $\alpha_{A}$ and $\alpha_{B}$ smaller than the corresponding market shares, and to have $\alpha_{A}$ almost equal to 0 .

4) Combined effect of market and spectrum share: The combined effect of market and spectrum shares is shown in Figures $2 \mathrm{~d}$ and 2e, reporting the core for scenarios S4 and $\mathbf{S 5}$, respecively. In scenario $\mathbf{S} 4$ the player with the minimum number of users is the only one owning a spectrum, while in scenario S5 the incumbent player is the only one owning a spectrum. The core of scenario S4 is very small and is not very sensitive to the value of $\delta$. The acceptable values of $\alpha_{A}$ are very small, never above 0.15 and $\alpha_{A}=0$ is acceptable. For $\delta=3, \alpha_{B}$ cannot rise above 0.5 : this means that the incumbent $C$ should pay most of the cost with a little help from $B$ so as they can both profit from the spectrum of $A$. Instead, in scenario S5, where the incumbent is the one providing the spectrum, $\alpha_{A}$ and $\alpha_{B}$ cannot be equal to 0 . For smaller values of $\delta$, neither $\alpha_{C}$ can be equal to 0 , as $A$ and $B$ do not find it profitable to cover for the whole expenses due to limited revenues. Instead, for $\delta=3, \alpha_{C}$ can be null, showing that $A$ and $B$ find it profitable to cover the whole expenses in order to profit of the spectrum of $C$.

\section{B. TU game results}

Many remarks can be extended to the TU case whose results are reported in Figure 3: for instance, the effect of $\delta$ is similar as for the NTU case, as the core enlarges with the increasing value of $\delta$. However, as revenues are assumed to be transferable, the value of $\alpha$ can also be negative, meaning that not only the player does not share the cost but it also receives part of the revenues of the other players. This of course depends on the spectrum and market shares. For scenario $\mathbf{S 1}$ with $\delta=3$, all the players can receive from others, although not simultaneously. Instead, in scenario S2 only players $B$ and $C$, that provide most of the spectrum, can receive revenues from the others, although not simultaneously: they are rewarded for providing spectrum by receiving more than their own revenue. In scenario $\mathbf{S 3}$ with $\delta \geq 1.5$, the players with the smallest number of users can be rewarded: for $\delta=3$ they can both and simultaneously receive utility from the incumbent $C$, which finds it profitable to give part of its revenues despite having to bear the whole expenses, as the increased available spectrum provides it with higher revenues. In scenarios $\mathbf{S 4}$ and $\mathbf{S 5}$ the only player providing the spectrum, $A$ in $\mathbf{S 4}$ and $C$ in $\mathbf{S 5}$, can be rewarded for high values of $\delta$. This is more accentuated in $\mathbf{S 4}$ where $A$ not only provides the overall spectrum but has also the smallest market share.

\section{Subcoalition analysis}

Table IV reports the stable coalitions for different ranges of the value of $\delta$ for all scenarios. When $\delta \leq 0.53$, no coalition finds it profitable to invest (denoted by the symbol -), whereas for $\delta \geq 0.58$ the core of the grand coalition is nonempty for all scenarios. Instead for instances with an empty core, stable subcoalitions are reported ${ }^{12}$.

\begin{tabular}{lccccc}
\hline & S1 & S2 & S3 & S4 & S5 \\
\hline$\delta \in[0.5,0.53]$ & - & - & - & - & - \\
$\delta=0.54$ & - & - & - & $\{A, C\}$ & $\{C\}$ \\
$\delta=0.55$ & - & $\{B, C\}$ & - & $\{A, C\}$ & $\{B, C\}$ \\
$\delta=0.56$ & $\{A, B, C\}$ & $\{A, B, C\}$ & $\{A, B, C\}$ & $\{A, C\}$ & $\{B, C\}$ \\
$\delta=0.57$ & $\{A, B, C\}$ & $\{A, B, C\}$ & $\{A, B, C\}$ & $\{A, B, C\}$ & $\{B, C\}$ \\
$\delta \in[0.58,3]$ & $\{A, B, C\}$ & $\{A, B, C\}$ & $\{A, B, C\}$ & $\{A, B, C\}$ & $\{A, B, C\}$ \\
\hline
\end{tabular}

TABLE IV: Stable coalitions for each scenario and value of $\delta$

We explore scenarios $\mathbf{S 4}$ and $\mathbf{S 5}$ when $\delta=0.55$, for which we study the core of each subcoalition of 2 MNOs (i.e., $\{A, B\}$, $\{A, C\}$ and $\{B, C\})^{13}$. We recall that in scenario $\mathbf{S 4}$ only $A$ has a spectrum license whereas in $\mathbf{S 5}$, only $C$. In case it is feasible for a coalition to invest (i.e., at least one of its members has a spectrum license), Table $\mathrm{V}$ reports whether the core is empty, otherwise, if nonempty, it indicates the range of stable cost fractions $\left(\alpha_{i}\right)$ and obtainable payoffs $\left(p_{i}\right)$ by each member MNO $i$.

In scenario $\mathbf{S 4}$, the core of the grand coalition is empty since $v(\{A, B, C\})<v(\{A, C\})$, i.e., $A$ and $C$ can be both better off in $\{A, C\}$. However, both $\{A, B\}$ and $\{A, C\}$ have a nonempty core. Since $B$ and $C$ cannot invest neither alone nor together, due to the lack of spectrum, both prefer collaborating with $A$. Instead, $A$ prefers $\{A, C\}$ to $\{A, B\}$ : if $C$ were to pay at least $86.26 \%$ of the cost of $\{A, C\}$ (which lies inside $C$ 's stable range of cost fractions and thus it is profitable (see Table $\mathrm{V})$ ), the payoff of $A$ from $\{A, C\}$, would be at least as large as the maximum payoff it can secure from $\{A, B\}$ (123153€), that is, if $B$ were to pay for all the $\{A, B\}$ cost. Consequently, $\{A, C\}$ will be created whereas $B$ will not invest at all. Such behavior is due to the very low value of $\delta$ (i.e., price per unit of service), which limits revenues and in turn the level of investment (i.e., number of activated BS) in order to be profitable. But since $\{A, B, C\}$ is more congested than $\{A, B\}$ and $\{A, C\}$ (no spectrum pooling gain since $B$ and $C$ do not contribute with spectrum) and requires more investment to lower the level of congestion, it is then less profitable. In turn, $A$ can better exploit its spectrum by collaborating with $C$ instead of $B$, since $C$ has the largest market share and thus can take up a larger fraction of their shared network cost.

For S5, the core is empty since the overall payoff of the grand coalition $(v(\{A, B, C\}))$ is strictly smaller than the overall payoff of any other subcoalition for which it is profitable to invest. In other words, $C$ is better off in any other subcoalition it can be part of than in $\{A, B, C\}$. Further, also $\{A, C\}$ has an empty core since $v(\{A, C\})<v(\{C\})$, that is, $C$ is better off by itself than collaborating with $A$, as $A$ can only cover a small portion of the $\{A, C\}$ cost, given its small market share.

\footnotetext{
${ }^{12}$ Notice that for all entries of the table in which the stable coalition consist of either one or two MNOs, the remaining MNOs do not invest at all.

${ }^{13}$ Similar observations can be drawn also for the other instances for which the grand coalition is not stable.
} 
Instead, $\{B, C\}$ has a nonempty core, thus $B$ and $C$ will build a shared network while $A$ will not invest at all. Although $C$ could be building its own network $(v(\{C\})>0)$, it prefers collaborating with $B$ which can pay up to $1 / 3$ of their shared network cost.

It can be observed that, in conditions of very low revenues, and in particular when there is no spectrum pooling gain, smaller coalitions and cooperation with bigger MNOs are preferred.

\begin{tabular}{lll}
\hline \multicolumn{3}{c}{$\mathbf{S 4}$} \\
\hline \multirow{2}{*}{$A, B$} & $\alpha_{\mathbf{A}}:[23.76,25.41] \%$ & $\mathbf{p}_{\mathbf{A}}:[0,123153] €$ \\
& $\alpha_{\mathbf{B}}:[74.59,76.24] \%$ & $\mathbf{p}_{\mathbf{B}}:[0,123153] €$ \\
\hline$\{A, C\}$ & $\alpha_{\mathbf{A}}:[12.46,14.59] \%$ & $\mathbf{p}_{\mathbf{A}}:[0,307490] €$ \\
& $\alpha_{\mathbf{C}}:[85.41,87.54] \%$ & $\mathbf{p}_{\mathbf{C}}:[0,307490] €$ \\
\hline$\{B, C\}$ & no spectrum license \\
\hline \multicolumn{3}{c}{$\mathbf{S 5}$} \\
\hline$\{A, B\}$ & no spectrum license \\
\hline$\{A, C\}$ & \multicolumn{3}{c}{ empty core $(v(\{A, C\})>0)$} \\
\hline$\{B, C\}$ & $\alpha_{\mathbf{B}}:[34.12,34.13] \%$ & $\mathbf{p}_{\mathbf{B}}:[0,1352] €$ \\
& $\alpha_{\mathbf{C}}:[65.87,65.88] \%$ & $\mathbf{p}_{\mathbf{C}}:[344749,346101] €$ \\
\hline
\end{tabular}

TABLE V: Core of subcoalitions for $\delta=0.55$ (same for the NTU and the TU games)

\section{Sharing gain}

Tables VI and VII summarize the gain of each MNO from joining the grand coalition relative to not sharing, that is, if they were to build individual networks. The values are calculated as $\frac{p_{i}-\bar{p}_{i}}{\bar{p}_{i}} 100 \%, \forall i \in O$, where $p_{i}$ is the payoff of MNO $i$ from the grand coalition according to the Nucleolus solution whereas $\bar{p}_{i}$ is its payoff when investing by itself. Notice that when it is either not profitable for an MNO to build its own network (i.e., its revenues do not cover its cost: e.g. MNO $A$ for $\delta=0.75$ ) or not feasible (the MNO has no spectrum license: e.g. MNOs $B$ and $C$ in $\mathbf{S 4}$ or $A$ and $C$ in S5), then $\bar{p}_{i}=0$. Such cases are represented by the $\infty$ symbol (the absolute gain is nevertheless finite).

As we calculate the sharing gain for the Nucleolus solution, which, by definition, tends to select a "fair" solution from the core, the NTU and TU models provide similar gains across all considered scenarios and cases ${ }^{14}$.

While the increase of $\delta$ increases the number of stable divisions of the grand coalition cost among the MNOs (illustrated by the increase of core size in Figures 2 and 3), Tables VI and VII indicate decreasing values of the relative gain as $\delta$ increases for all scenarios but S5. This shows how sharing is more beneficial when low revenues significantly limit the level of investment in network infrastructure an MNO can undertake by itself. Nevertheless, sharing remains profitable

\footnotetext{
${ }^{14}$ For scenario $\mathbf{S 4}, \delta=3$, which represents an extreme case, the nucleolus solutions of the two games are however significantly different: the relative gain of MNO $A$ under the TU model is one order of magnitude larger compared to the NTU one. Such behavior was also reflected in the core size being significantly larger in case of the TU game w.r.t. to the NTU one (Figures 2 and 3).
}

even for higher values of $\delta$, as MNOs still benefit from a larger pool of spectrum resources and cost sharing.

As expected, identical MNOs obtain equal gains (scenario S1). Scenario $\mathbf{S 2}$ shows the benefit of spectrum pooling: the smaller the MNO spectrum share, the more it benefits from the grand coalition, despite having to pay for a larger fraction of its infrastructure cost. Instead, scenario $\mathbf{S 3}$ shows how MNOs with smaller market shares, which find it more difficult to face the network upfront cost by themselves, incur larger gains from cooperation. In particular, for scenarios $\mathbf{S 4}$ and S5, since there is no spectrum pooling gain (only one MNO has a spectrum license), the relative gain is much smaller compared the other scenarios, especially for MNO $C$ (scenario S5), which has less difficulties covering its network cost given its large market share (as opposed to $A$ in scenario $\mathbf{S 4}$, that, despite owning a spectrum license, has limited revenues given its small share of users). Contrarily to the other scenarios, in S5, sharing becomes more beneficial for $C$ as $\delta$ increases, as $A$ and $B$ can afford to cover a larger fraction of the grand coalition cost.

\begin{tabular}{|c|c|c|c|c|}
\hline & & $A$ & $B$ & $C$ \\
\hline \multirow{3}{*}{ S1 } & $\delta=0.75$ & $2052.67 \%$ & $2052.67 \%$ & $2052.67 \%$ \\
\hline & $\delta=1.5$ & $290.99 \%$ & $290.99 \%$ & $290.99 \%$ \\
\hline & $\delta=3$ & $254.94 \%$ & $254.94 \%$ & $254.94 \%$ \\
\hline \multirow{3}{*}{ S2 } & $\delta=0.75$ & $\infty$ & $2179.71 \%$ & $206.64 \%$ \\
\hline & $\delta=1.5$ & $9374.79 \%$ & $317.69 \%$ & $151.37 \%$ \\
\hline & $\delta=3$ & $1459.48 \%$ & $275.62 \%$ & $146.01 \%$ \\
\hline \multirow{3}{*}{ S3 } & $\delta=0.75$ & $61995.21 \%$ & $1998.13 \%$ & $901.87 \%$ \\
\hline & $\delta=1.5$ & $833.41 \%$ & $329.98 \%$ & $216.03 \%$ \\
\hline & $\delta=3$ & $433.20 \%$ & $298.55 \%$ & $205.77 \%$ \\
\hline \multirow{3}{*}{ S4 } & $\delta=0.75$ & $431.54 \%$ & $\infty$ & $\infty$ \\
\hline & $\delta=1.5$ & $109.35 \%$ & $\infty$ & $\infty$ \\
\hline & $\delta=3$ & $50.82 \%$ & $\infty$ & $\infty$ \\
\hline \multirow{3}{*}{ S5 } & $\delta=0.75$ & $\infty$ & $\infty$ & $23.22 \%$ \\
\hline & $\delta=1.5$ & $\infty$ & $\infty$ & $26.74 \%$ \\
\hline & $\delta=3$ & $\infty$ & $\infty$ & $32.58 \%$ \\
\hline
\end{tabular}

TABLE VI: NTU game: sharing gain

\begin{tabular}{clccc}
\hline & & $A$ & $B$ & $C$ \\
\hline \multirow{3}{*}{ S1 } & $\delta=0.75$ & $2052.67 \%$ & $2052.67 \%$ & $2052.67 \%$ \\
& $\delta=1.5$ & $290.99 \%$ & $290.99 \%$ & $290.99 \%$ \\
& $\delta=3$ & $254.94 \%$ & $254.94 \%$ & $254.94 \%$ \\
\hline \multirow{3}{*}{ S2 } & $\delta=0.75$ & $\infty$ & $2176.61 \%$ & $207.15 \%$ \\
& $\delta=1.5$ & $9378.69 \%$ & $342.27 \%$ & $141.57 \%$ \\
& $\delta=3$ & $1428.44 \%$ & $262.00 \%$ & $154.14 \%$ \\
\hline \multirow{3}{*}{ S3 } & $\delta=0.75$ & $61817.36 \%$ & $2010.21 \%$ & $899.36 \%$ \\
& $\delta=1.5$ & $918.79 \%$ & $314.99 \%$ & $210.30 \%$ \\
& $\delta=3$ & $703.23 \%$ & $265.31 \%$ & $177.26 \%$ \\
\hline \multirow{3}{*}{ S4 } & $\delta=0.75$ & $448.76 \%$ & $\infty$ & $\infty$ \\
& $\delta=1.5$ & $456.01 \%$ & $\infty$ & $\infty$ \\
& $\delta=3$ & $574.99 \%$ & $\infty$ & $\infty$ \\
\hline \multirow{3}{*}{ S5 } & $\delta=0.75$ & $\infty$ & $\infty$ & $23.10 \%$ \\
& $\delta=1.5$ & $\infty$ & $\infty$ & $39.06 \%$ \\
& $\delta=3$ & $\infty$ & $\infty$ & $38.45 \%$ \\
\hline
\end{tabular}

TABLE VII: TU game: sharing gain 


\section{DISCUSSION}

This work has targeted sharing of $4 \mathrm{G}$ small cells and proposed a particular pricing and cost model. However, the proposed game models are useful tools to study other technologies as well (e.g., 3G/4G macro cells) and/or different pricing and cost models. In the following, we discuss the impact of some of the assumptions made and the applicability of the models to alternative settings.

- As investment in network infrastructure and spectrum availability are both key to improving the service level (i.e, data rate here), the proposed pricing model aims at translating the two into revenues. Since nowdays MNOs struggle with monetizing their investments (either in infrastructure of spectrum licenses), roughly speaking, the considered revenues would represent an overestimation. Nevertheless, by considering a wide range of such revenues, we were able to see their impact on the stable coalitions and their corresponding cost divisions. We also note that it is outside the scope of this work to investigate pricing models in line with those in the market, such as bundles of different types of services and data usage caps. In these lines, as the churn rate is determined by marketing strategies rather than technical factors, we do not address the user migration among MNOs.

- The considered cost model accounts for the main upfront and operational cost terms related to the radio equipments and for the backhauling cost. Although a more realistic backhauling cost model could be used instead, our goal was to overestimate its cost, in order to have a more significant sharing tradeoff, that is, between benefiting from larger spectrum resources when in a larger coalition but incurring a higher cost to which amounts also a higher level of congestion. The backhaul optimization is also outside the scope of this work.

- We do not account for the spectrum license cost since we assume MNOs have purchased the spectrum license individually and prior to entering a sharing agreement. The amount of spectrum available to a coalition depends then on its members contribution, and thus is not part of its strategy, unlike the investment in network infrastructure. The models can nevertheless take into account such cost as follows: Let $\hat{c}_{i}$ be the spectrum license cost of MNO $i \in O$, representing an upfront cost. It is then only profitable for an MNO to be in the grand coalition if its allocated payoff can cover $\hat{c}_{i}$, that is, $p_{i} \geq \hat{c}_{i}, \forall i \in O$. Such constraint translates into an upper bound on the fraction of cost the MNO would be willing to pay to be in the grand coalition:

$p_{i} \geq \hat{c}_{i} \Longrightarrow \tilde{r}_{O}^{i}-\alpha_{O}^{i} \tilde{c}_{O} \geq \hat{c}_{i} \Longrightarrow \alpha_{O}^{i} \leq \frac{\tilde{r}_{O}^{i}-\hat{c}_{i}}{\tilde{c}_{O}}, \forall i \in O$.

If $\left(\tilde{r}_{O}^{i}-\hat{c}_{i}\right) / \tilde{c}_{O}<1$, the constraints would reduce the set of feasible payoffs of the proposed models. Further, if $\sum_{i \in O}\left(\tilde{r}_{O}^{i}-\hat{c}_{i}\right) / \tilde{c}_{O}<1$, then the grand coalition would not be created. If the core was empty, the constraints would similarly be extended to subcoalitions.
We did not carry out such analysis since a spectrum license cost depends on several factors such as the spectrum auction time and place and the spectrum band. However, assuming the spectrum license cost is proportional to its amount of spectrum, the larger the spectrum provided by an MNO, the smaller the cost fraction it would pay to be in a coalition. This behavior is indirectly observed even without explicitly taking into account the spectrum license cost, as MNOs contributing with a larger spectrum share tend to pay less than the others.

\section{CONCLUSIONS}

This work investigates the problem of RAN and spectrum sharing in $4 \mathrm{G}$ networks for a scenario in which MNOs with fixed market and spectrum shares plan to upgrade their existing RAN by deploying small cell BSs. Each MNO weighs between deploying an individual network or enter a sharing agreement with other MNOs and thus build a shared network. We assume that when MNOs build a shared network, they will aggregate their spectrum resources. A generic mobile data pricing model is introduced to determine revenues incurred by an MNO from each possible coalition (sharing agreement). We propose two cooperative game models to address the problem: if MNOs in a coalition agree to share its cost but keep their individual revenues, the problem is formalized as a non-transferable utility cooperative game; if MNOs would be willing to give away also part of their individual revenues to be in a coalition, a transferable utility game is proposed instead. The core and nucleolus solution concepts are leveraged to determine stable cost divisions.

The proposed models are investigated for several instances with different network and economic settings aiming to represent realistic scenarios. For the vast majority of the considered instances, MNOs are better off building a unique shared RAN than creating sub-coalitions or building individual RANs due to the combined gain from spectrum aggregation and cost reduction from sharing the network infrastructure. The cost division of the shared network infrastructure that guarantees stability depends both on network and economic inputs: MNOs with a larger customer base should be accounted for a larger fraction of the cost; instead, MNOs contributing with a larger spectrum portion are "rewarded" by a lower cost fraction. In particular, MNOs which provide the largest spectrum portion are not only exempted from the network infrastructure cost but can also receive part of the other MNOs revenues. Dividing the cost based on the market share does not always guarantee stability whereas the stable cost division selected by the nucleolus, which in turn accounts also for the MNOs spectrum contribute, makes a better candidate for a cost division policy.

The models we propose here are generic instruments for addressing the problem of network sharing from a strategic perspective as they can accommodate for alternative technologies and/or pricing models and cost functions.

\section{ACKNOWLEDGMENT}

The present work has been partially supported by the EU project ACT5G (H2020 MSCA-ITN, project no. 643002). 


\section{REFERENCES}

[1] CISCO, "Cisco Visual Networking Index: Global mobile data traffic forecast update, 2015-2020 white paper," http: //www.cisco.com/c/en/us/solutions/collateral/service-provider/visualnetworking-index-vni/mobile-white-paper-c11-520862.html, [Online; Accessed: 2016-02-27]

[2] W. Hare, "Western European mobile operators must take action in the face of a deteriorating retail revenue outlook," http://www.analysysmason.com/Research/Content/Comments/WesternEurope-forecast-comment-Jan2014-RDDF0/, 2014, [Online; Accessed 2016-04-07].

[3] GSMA, "Spectrum for new entrants, lessons learned," https://gsmaintelligence.com/research/?file= 3f4ec58d593cdd88d2a7e71995e82733\&download, [Online; Accessed: 2016-02-21].

[4] E. Heinrich, "Telecom companies count $\$ 386$ billion in lost revenue to Skype, WhatsApp, others," http://fortune.com/2014/06/23/telecomcompanies-count-386-billion-in-lost-revenue-to-skype-whatsappothers/, 2014, [Online; Accessed 2016-04-07].

[5] GSMA, "Mobile infrastructure sharing," http://www.gsma.com/ publicpolicy/wp-content/uploads/2012/09/Mobile-Infrastructuresharing.pdf, 2008, [Online; Accessed 2016-04-10].

[6] Telenor, "Telenor and Tele2 to build joint 4G network in Sweden," https://www.telenor.com/media/press-releases/2009/telenor-and-tele2to-build-joint-4g-network-in-sweden/, 2009, [Online; Accessed: 2016-02-27].

[7] J. Markendahl, "Shared networks lessons learned 2000-2010. What differences can we observe in Sweden?" https://www.kth.se/social/ upload/50a1730df2765431108bc382/WIDE\%20Nov\%2012\%20$\% 20$ Network\%20sharing\%202000\%20and\%202010.pdf, [Online; Accessed: 2016-04-10].

[8] D.-E. Meddour, T. Rasheed, and Y. Gourhant, "On the role of infrastructure sharing for mobile network operators in emerging markets," Computer Networks, vol. 55, no. 7, pp. 1576-1591, 2011.

[9] BEREC/RSPG, "Joint BEREC/RSPG report on Infrastructure and spectrum sharing in mobile/wireless networks," http://rspg-spectrum.eu/wpcontent/uploads/2013/05/rspg11-374_final_joint_rspg_berec_report.pdf, [Online; Accessed: 2016-02-27].

[10] Industry Canada, "Framework for mandatory roaming and antenna tower and site sharing," http://www.ic.gc.ca/eic/site/smt-gst.nsf/eng/h_ sf10290.html, [Online; Accessed: 2016-02-21].

[11] ITU, "Mobile Infrastructure Sharing: Trends in Latin America," https://www.itu.int/en/ITU-D/Regulatory-Market/Documents/ CostaRica/Presentations/Session8_Daniel\%20Leza\%20-\%20Mobile\% 20Infrastructure\%20Sharing\%20-\%2012\%20March\%202014.pdf, [Online; Accessed: 2016-02-21].

[12] 3GPP TS 22.951, "Service aspects and requirements for network sharing," v.11.0.0, 2012

[13] 3GPP, "Carrier Aggregation explained," http://www.3gpp.org/ technologies/keywords-acronyms/101-carrier-aggregation-explained, [Online; Accessed: 2016-02-03].

[14] Global Mobile Suppliers Association (GSA), "LTE-Advanced Carrier Aggregation deployments: peak speeds report," http: //gsacom.com/paper/lte-advanced-carrier-aggregation-deploymentspeak-speeds-report-116-networks-launched/, [Online; Accessed: 2016-02-21].

[15] S. Valentin, W. Jamil, and O. Aydin, "Extending generalized processor sharing for multi-operator scheduling in cellular networks," in Wireless Communications and Mobile Computing Conference (IWCMC), 2013 9th International. IEEE, 2013, pp. 485-490.

[16] I. Malanchini, S. Valentin, and O. Aydin, "Generalized resource sharing for multiple operators in cellular wireless networks," in Wireless Communications and Mobile Computing Conference (IWCMC), 2014 International. IEEE, 2014, pp. 803-808.

[17] A. Gudipati, D. Perry, L. E. Li, and S. Katti, "SoftRAN: Software defined radio access network," in Proceedings of the second ACM SIGCOMM workshop on Hot topics in software defined networking. ACM, 2013, pp. 25-30.

[18] A. Gudipati, L. E. Li, and S. Katti, "RadioVisor: A slicing plane for Radio Access Networks," in Proceedings of the Third Workshop on Hot Topics in Software Defined Networking, ser. HotSDN '14, 2014, pp. 237-238.

[19] X. Costa-Pérez, J. Swetina, T. Guo, R. Mahindra, and S. Rangarajan, "Radio access network virtualization for future mobile carrier networks," IEEE Communications Magazine, vol. 51, no. 7, 2013.
[20] S. L. Hew and L. B. White, "Cooperative resource allocation games in shared networks: Symmetric and asymmetric fair bargaining models," IEEE Transactions on Wirelss Communications, vol. 7, no. 11, 2008.

[21] M. A. Khan, A. C. Toker, C. Troung, F. Sivrikaya, and S. Albayrak, "Cooperative game theoretic approach to integrated bandwidth sharing and allocation," International Conference on Game Theory for Networks, pp. 1-9, 2009 .

[22] F. Teng, D. Guo, and M.-L. Honig, "Sharing of unlicensed spectrum by strategic operators," in GlobalSIP Symposium on Game Theory for Signal Processing and Communications, 2014.

[23] Y. Xiao, C. Yuen, P. Di Francesco, and L. A. DaSilva, "Dynamic spectrum scheduling for carrier aggregation: A game theoretic approach," in Communications (ICC), 2013 IEEE International Conference on. IEEE, 2013, pp. 2672-2676.

[24] Y. Xiao, Z. Han, C. Yuen, and L. A. DaSilva, "Carrier aggregation between operators in next generation cellular networks: A stable roommate market," IEEE Transactions on Wireless Communications, vol. 15, no. 1, pp. 633-650, 2016.

[25] Y.-T. Lin, H. Tembine, and K.-C. Chen, "Inter-operator spectrum sharing in future cellular systems," in Global Communications Conference (GLOBECOM), 2012 IEEE. IEEE, 2012, pp. 2597-2602.

[26] C. Liang and F. R. Yu, "Wireless network virtualization: A survey, some research issues and challenges," Communications Surveys \& Tutorials, IEEE, vol. 17, no. 1, pp. 358-380, 2015.

[27] M. Yang, Y. Li, D. Jin, L. Zeng, X. Wu, and A. V. Vasilakos, "Softwaredefined and virtualized future mobile and wireless networks: A survey," Mobile Networks and Applications, vol. 20, no. 1, pp. 4-18, 2015.

[28] L. A. DaSilva, J. Kibilda, P. DiFrancesco, T. K. Forde, and L. E. Doyle, "Customized services over virtual wireless networks: The path towards networks without borders," in Future Network and Mobile Summit (FutureNetworkSummit), 2013. IEEE, 2013, pp. 1-10.

[29] L. Doyle, J. Kibilda, T. K. Forde, and L. DaSilva, "Spectrum without bounds, networks without borders," Proceedings of the IEEE, vol. 102, no. 3, pp. 351-365, 2014

[30] ITU, "Spectrum sharing," http://www.ictregulationtoolkit.org/5.4, [Online; Accessed: 2016-04-10].

[31] P. Di Francesco, F. Malandrino, T. Forde, and L. DaSilva, "A sharing-and competition-aware framework for cellular network evolution planning," IEEE Transactions on Cognitive Communications and Networking, pp. 230-243, 2015

[32] J. Kibilda, P. Di Francesco, F. Malandrino, and L. A. DaSilva, "Infrastructure and spectrum sharing trade-offs in mobile networks," in $D y$ namic Spectrum Access Networks (DySPAN), 2015 IEEE International Symposium on. IEEE, 2015, pp. 348-357.

[33] SAPHYRE, "Business models, cost analysis and advises for spectrum policy and regulation for scenario III (full sharing)," http://www.saphyre. eu/intranet/deliverables/archive_sent/d5.5.pdf, 2013, [Online; Accessed: 2016-02-03].

[34] F. Offergelt, F. Berkers, and G. Hendrix, "If you can't beat'em, join'em cooperative and non-cooperative games in network sharing," in Intelligence in Next Generation Networks (ICIN), 2011 15th International Conference on. IEEE, 2011, pp. 196-201.

[35] F. Offergelt, "Analysing sharing scenarios for mobile network operators using game theory," Master's thesis, Universiteit Leiden, 2011.

[36] L. Cano, A. Capone, G. Carello, and M. Cesana, "Evaluating the performance of infrastructure sharing in mobile radio networks,", in IEEE International Conference on Communications (ICC), London UK, 2015, pp. 1-6.

[37] L. Cano, A. Capone, G. Carello, M. Cesana, and M. Passacantando, "A Non-cooperative Game Approach for RAN and Spectrum Sharing in Mobile Radio Networks," in European Wireless 2016; 22th European Wireless Conference. VDE, 2016, pp. 1-6.

[38] K. Johansson, "Cost effective deployment strategies for heterogenous wireless networks," KTH, 2007.

[39] K. Johansson, A. Furuskar, P. Karlsson, and J. Zander, "Relation between base station characteristics and cost structure in cellular systems," IEEE International Symposium on Personal, Indoor and Mobile Radio Communications (PIMRC), vol. 4, pp. 2627-2631, 2004.

[40] K. Johansson, J. Zander, and A. Furuskar, "Modelling the cost of heterogeneous wireless access networks," International Journal of Mobile Network Design and Innovation, vol. 2, no. 1, pp. 58-66, 2007.

[41] Z. Frias and J. Pérez, "Techno-economic analysis of femtocell deployment in long-term evolution networks," EURASIP journal on wireless communications and networking, vol. 2012, no. 1, pp. 1-15, 2012.

[42] Green Touch - Mobile Communication WG, "Architecture Doc 2: Reference scenarios," http://www.greentouch.org/, 2013. 
[43] OECD, "Wireless market structures and network sharing," http://www.oecd-ilibrary.org/docserver/download/5jxt46dz19r2. pdf? expires $=1459895381 \&$ id=id\&accname $=$ guest $\&$ checksum $=$ 2D7136DFD5898C6E6D767714D7D2FE67, 2014, [Online; Accessed 2016-03-30].

[44] Newswire, "Rogers and Videotron to build-out expanded LTE network in Quebec and Ottawa," http://www.newswire.ca/news-releases/rogersand-videotron-to-build-out-expanded-lte-network-in-quebec-andottawa-512486471.html, [Online; Accessed: 2016-02-21].

[45] C. Bouras, V. Kokkinos, and A. Papazois, "Financing and pricing small cells in next generation mobile networks," in 12th International Conference on Wired and Wireless Internet Communications (WWIC 2014), Paris France, May 2014, pp. 1-14.

[46] M. Nakayama, "A note on a generalization of the nucleolus to games without sidepayments," Internat. J. Game Theory, vol. 12, no. 2, pp. 115-122, 1983

[47] D. Schmeidler, "The nucleolus of a characteristic function game," SIAM J. Appl. Math., vol. 17, pp. 1163-1170, 1969.

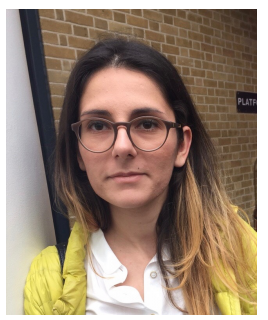

Lorela Cano is a PhD student at DEIB, Politecnico di Milano. Her main research interests are in the area of techno-economic characterization of infrastructure sharing in networks based on game theoretical models.

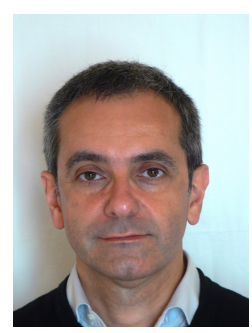

Antonio Capone is Full Professor at Politecnico di Milano (Technical University of Milan), where he is the director of the ANTLab. His expertise is on networking and his main research activities include radio resource management in wireless networks, traffic management in software defined networks, network planning and optimization. On these topics he has published more than 200 peer-reviewed. He serves in the TPC of major conferences in networking, he is editor of IEEE Trans. on Mobile Computing, Computer Networks, and Computer Communications, and he was editor of ACM/IEEE Trans. on Networking from 2010 to 2014

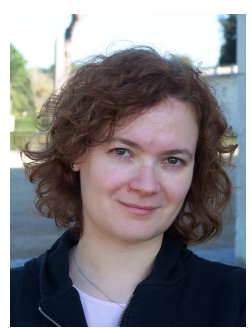

Giuliana Carello is assistant professor in the Operation Research Group of DEIB (Dipartimento di Elettronica, Informazione e Bioingegneria) of Politecnico di Milano since 2005. Her research work interests are exact and heuristic optimization approaches, applied to integer and binary variable problems. Her research is mainly devoted to real life applications, such as telecommunication networks or health care management. She published peer-reviewed papers in international journals and conference proceedings.

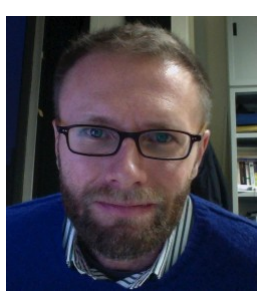

Matteo Cesana is currently an Associate Professor with the Dipartimento di Elettronica, Informazione e Bioingegneria of the Politecnico di Milano, Italy. He received his MS degree in Telecommunications Engineering and his Ph.D. degree in Information Engineering from Politecnico di Milano in July 2000 and in September 2004, respectively. From September 2002 to March 2003 he was a visiting researcher at the Computer Science Department of the University of California in Los Angeles (UCLA). His research activities are in the field of design, optimization and performance evaluation of wireless networks with a specific focus on wireless sensor networks and cognitive radio networks. Dr. Cesana is an Associate Editor of the Ad Hoc Networks Journal.

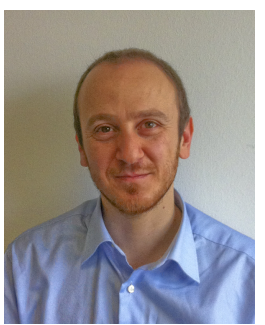

Mauro Passacantando received the M.S. and the $\mathrm{Ph} . \mathrm{D}$. degrees in Mathematics from the University of Pisa (Italy) in 2000 and 2005, respectively. From 2002 to 2012 he was an Assistant Professor at the Department of Applied Mathematics of University of Pisa. He is currently an Assistant Professor of Operations Research at the Department of Computer Science of University of Pisa. He published more than 40 peer-reviewed papers in books, conference proceedings and international journals. His research is mainly devoted to variational inequalities and equilibrium problems, concerning both theory and algorithms. In the last years, he worked on non-cooperative game theoretic approaches to the service provisioning problem in cloud and multi-cloud systems. 